\title{
Nanoproteomics for nanomedicine
}

"Label-free NAPPA technology, in combination with protein nanobiocrystallography and its possible future development using anodic porous alumina along with a cell-free expression system ... appear to form a single approach capable of effectively solving the numerous problems still present in medical diagnosis and therapy."
A new integrated approach for medical diagnostics and therapy, termed nanoproteomics, is emerging from the interplay between protein nanocrystallography and nucleic acid programmable protein arrays (NAPPAs) on a previously unseen scale.

Nanogenomics [1,2] and nanoproteomics [3,4] allow us to study and compare the huge amount of genes and proteins involved in the cell-cycle progression of human T lymphocytes [5] and in its transformation in lymphoma [6]. However, nanogenomics suffers many pitfalls that only functional proteomics, in the form of NAPPA, can overcome, probing the native in situ protein-protein interaction with unique sensitivity (Figure 1). This facilitates the identification of the key proteins involved in the control of cell function [Spera R, Sivozhelezov V, Badino F, Labaer J, Nicolini C: Maldi TOF detection of NAPPa, Manuscript Submitted] [3-8] and enables their further characterization by structural proteomics at the atomic level using synchrotron radiation [9-12]. It is the combination of protein nanocrystallography and NAPPA technologies that provides the focus of this article.

Most protein structures are now determined in synchrotrons, with $\mathrm{x}$-ray crystallography being the most widely used method for protein structure determination [9-13]. The synchrotron radiation facility provides a well-tuned size and wavelength of x-ray beams [14,15]. However, successful structure determination and refinement requires higher doses to measure statistically significant data that are important to determine protein structure and, thus, structural information is lost while collecting significant sets of data. Free radicals, formed by photon absorption (primary effect), move throughout the crystal (secondary effect), leaving fingerprints on proteins [16,17]. This problem in $\mathrm{x}$-ray crystallography takes the form of radiation damage to protein crystals and appears highly reduced in the Langmuir-Blodgett (LB) nanotemplate protein crystal [18]. Recently, many techniques, such as cryocooling, free radical scavenging and beam defocusing, have been introduced to reduce radiation damage. However, issues still persist and become highly problematic in microcrystallography $[14,15,19]$, which is at the frontiers of protein crystallography.

\section{New label-free developments in NAPPA microarrays}

Label-free protein-protein interactions were recently monitored [4] using self-assembling protein NAPPA microarrays and atomic force microscopy (AFM) [7], nanogravimetry [8], mass spectrometry [Spera R, Sivozhelezov V, Badino F, Labaer J, Nicolini C: Maldi TOF DETECTION OF NAPPA, MANUSCRipt Submitted] and anodic porous alumina [20] in an attempt to monitor the proteome alteration associated with cell proliferation, differentiation and neoplastic transformation.

In separate fluorescent-labeling NAPPA experiments [6] utilizing our DNA analyser (DNASER) $[1,21,22]$, a comparison was carried out with the well-established NAPPA fluorescence analysis [3], confirming the reliability of the above NAPPA label-free approaches [23]. Furthermore, very recent experimental activities have successfully demonstrated simple NAPPA printing on anodic porous alumina in a high-throughput format, with the ability to rapidly exchange fluids [20].

Prototypes are already built and are in the optimizing and validation phase. With regard to specific applications, the AFM and quartz crystal microbalance label-free technique could be utilized to investigate the kinetics of protein-protein interactions and to optimize NAPPA printing as a function of $\mathrm{pH}$, temperature, reagent composition and concentration. Such a technique could also be used to optimize the conditions for cellfree expression of a given protein (see later). Mass

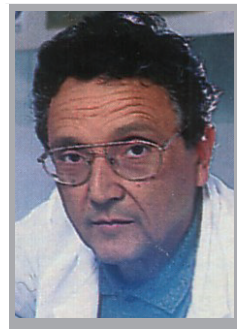

Claudio Nicolini

Author for correspondence:

Nanoworld Institute, Fondazione ELBA \& CIRSDNNOB, University of Genova,

Corso Europa 30, 16132 Genoa, Italy

Tel.: + 3901035338217

Fax: +3901035338215

manuscript@ibf.unige.it

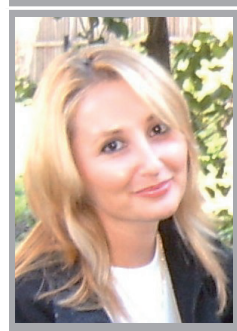

Eugenia Pechkova

Nanoworld Institute, Fondazione ELBA \& CIRSDNNOB University of Genova, Corso Europa 30, 16132 Genoa, Italy 


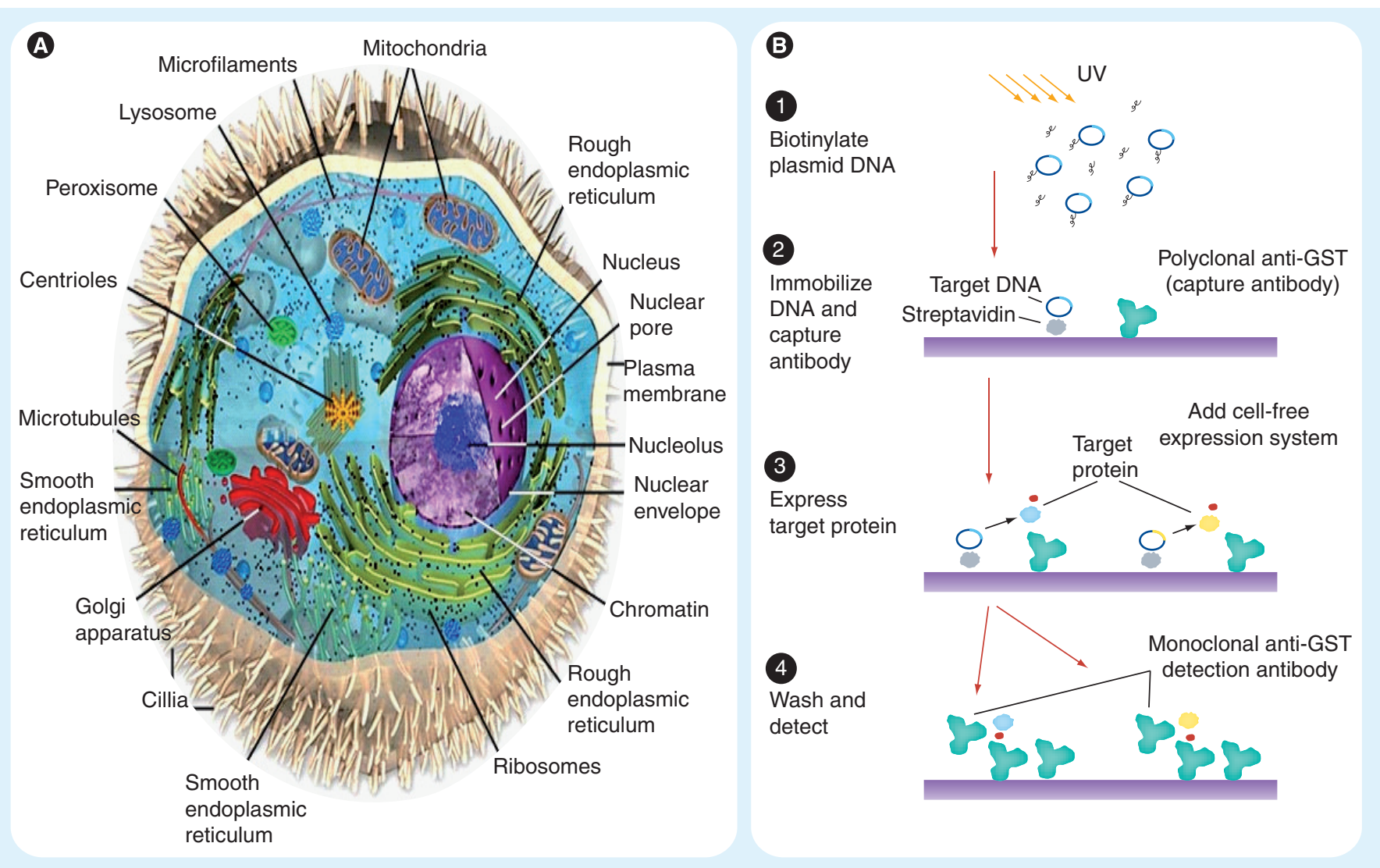

Figure 1. Determination of protein-protein interaction via nucleic acid programmable protein array. Protein-protein interactions representing key elements of cell function (A) can be assayed at large scale with the nucleic acid programmable protein array approach (B). GST: Glutathione S-transerase.

Reproduced with permission from [4].

spectrometry has made the most progress in terms of coupling a label-free method to NAPPAs, clearly demonstrating the usefulness of MS spectra for the analysis of protein-protein interactions [Spera R, Sivozhelezov V, Badino F, Labaer J, Nicolini C: Maldi TOF detection of NAPPA, Manuscript Submitted]. In this case, the goal was to demonstrate that we can identify proteins, in particular proteins that bind to the target proteins on the array.

\section{New protein crystal properties using LB \& synchrotron radiation}

Highly ordered, well-diffracting and radiationstable crystals remain important for successful and significant data collection. Crystals grown by LB-based methods [24] have previously proved to be better in terms of data quality and resolution [25]. Radiation stability in terms of reflection intensities have also been revealed for lysozyme crystals based on LB-based methods [18], such as reduction of diffractive power and resolution, breaking of specific residues, increase of mosaicity and overall B-factor and crystal unit cell volume [12,16]. Recently, a detailed study of numerous protein crystals grown by LB- and classical-based methods at the European Synchrotron Radiation Facility (ESRF) in Grenoble (France) demonstrated that the effects of differential radiation damage before and after administration of a high x-ray

Table 1. Proteins uniquely crystallized by Langmuir-Blodgett nanotemplates with
3D atomic structure parameters determined by microperoxidase.
\begin{tabular}{llr} 
Proteins & Average size $(\boldsymbol{\mu m})$ & Ref. \\
Cytochrome P450scC & $5 \times 5 \times 5$ & {$[28]$} \\
Human kinase CK2 $\alpha$ & $50 \times 10 \times 10$ & {$[1]$} \\
IF2 $\alpha$ l & $20 \times 10 \times 10$ & {$[9]$} \\
IF2 $\beta$ & $50 \times 10 \times 10$ & {$[9]$} \\
GroEL & $60 \times 10 \times 20$ & {$[29]$} \\
\hline
\end{tabular}




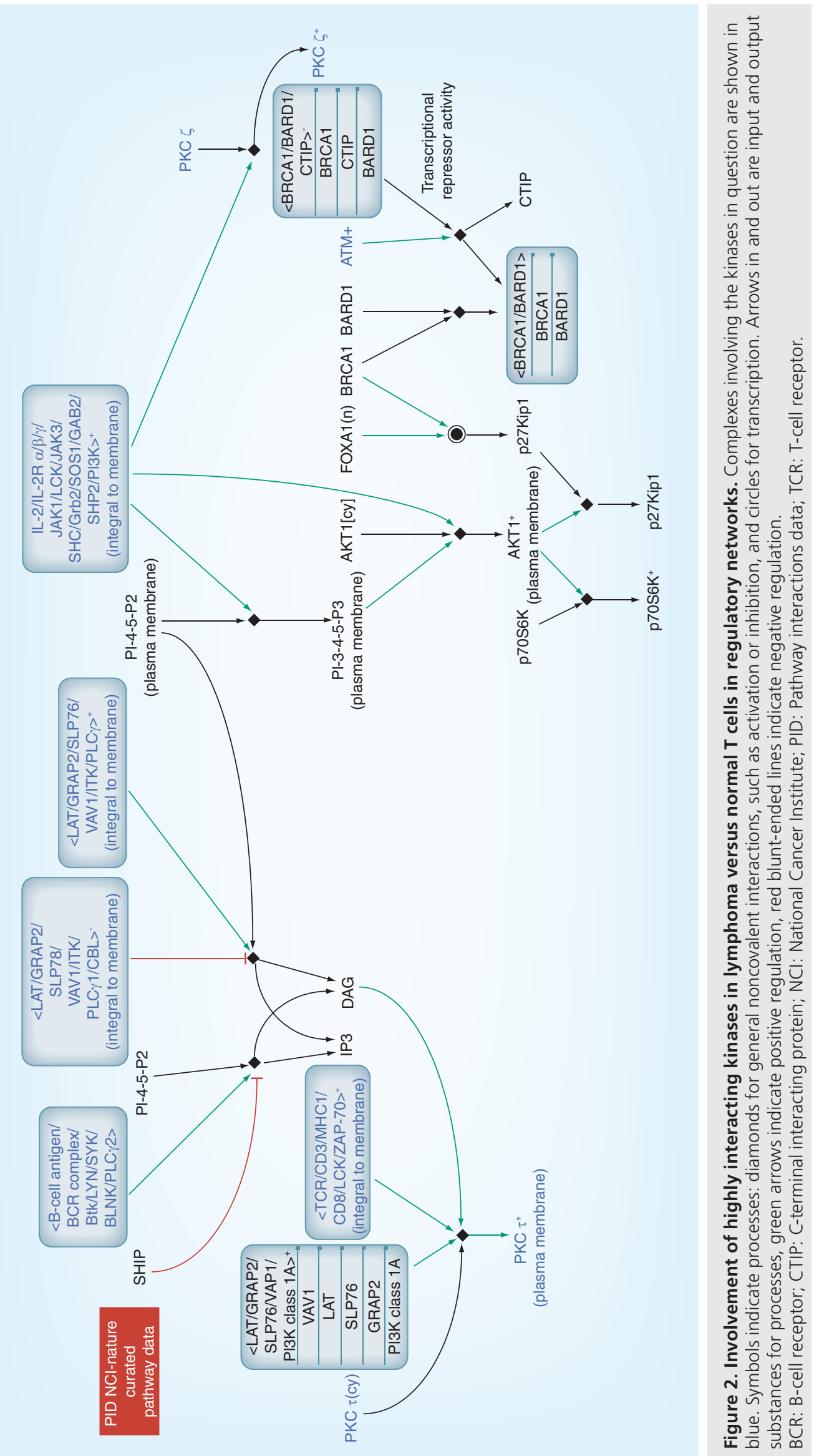


dose (burning) are less pronounced in LB crystals [26]. Extension of this study to the nanoscale is presented here to identify unexpected crystal topography probed by AFM [27] and laser microscopy [28]. Domain organization becomes apparent by a combination of AFM and laser technologies [16].

Langmuir-Blodgett nanofilms placed over cover glass slides were used as templates for crystallization [9-11], and the emerging protein crystals demonstrate clear domain structures [16]. They are stable under high-intensity radiation $[18,24,26]$ and can be formed for a wide range of important protein systems that are yet to be solved (Table 1). Finally, for the first time, microcrystallography of submicron laser-cut LB lysozyme crystals has been demonstrated to be capable of revealing atomic structures of ultrasmall protein crystals at a resolution of $2.0 \AA[28]$.

\section{Protein expression \& cancer}

A few key examples of possible biomedical applications of NAPPAs are given in Figure 2 for the study of normal T-lymphocyte proliferation and of their transformation in lymphoma. This makes clear the differentiation and advancement of nanoproteomics with respect to classical proteomics, which is defined as the identification, isolation and characterization of cell

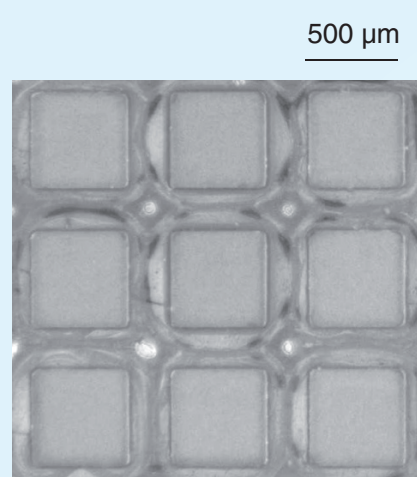

APA
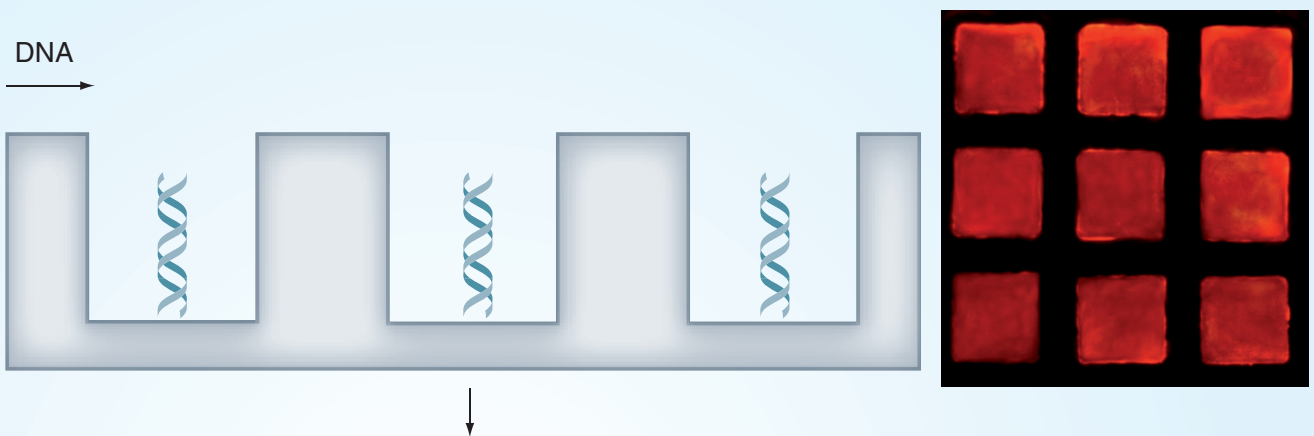

Protein cell-free expression

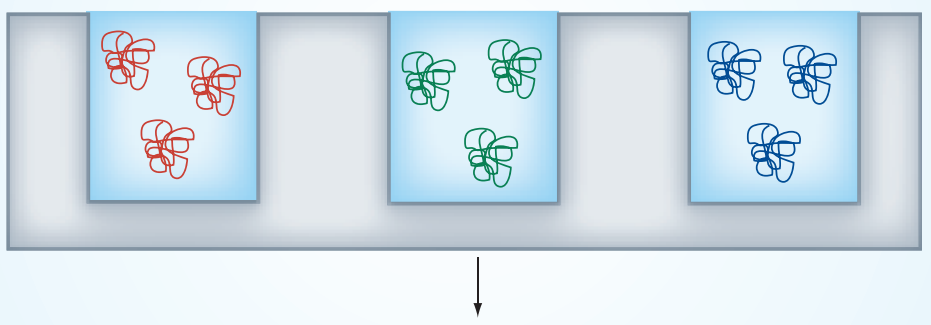

Membrane

High-throughput microdialysis
Salt solution

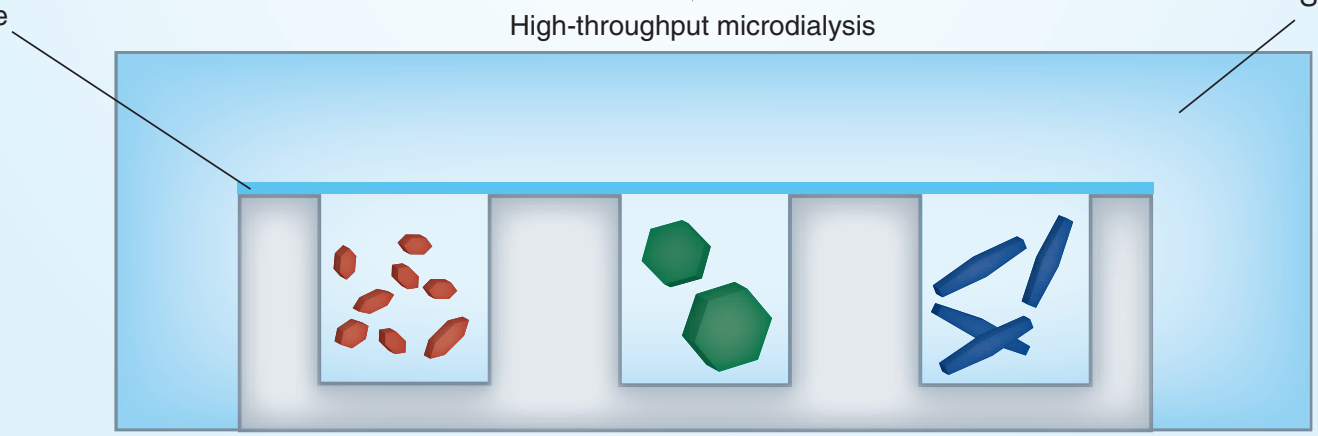

Figure 3. The future anodic porous alumina-nucleic acid programmable protein array cell-free based nanobiocrystallography, where anodic porous alumina channels can be used as the very small crystallization wells for the protein and precipitate solution, which can be covered with the layer of the silicon oils mixture (for the crystallization under oil) or with the microdialysis membrane (for the microdialysis crystallization, where the overall system is immersed in the precipitant solution).

APA: Anodic porous alumina. 
proteins within the framework of traditional current biomedicine. However, the mechanisms of cell transformation and cell-cycle progression remain largely obscure and need further exploration for the optimal control of cancer, which is still the most fatal disease in humans worldwide [6].

\section{Drug discovery}

The main conclusion of the earlier reported experiment at the submicron protein crystal is that a high-resolution diffraction pattern can be obtained from less than $1 \mu^{3}$ crystal volume, corresponding to approximately $2 \times 10^{7}$ unit cells. Despite the high irradiation dose in this case, it was possible to obtain an excellent high-resolution map and it could be concluded from the individual atomic B-factor patterns that there was no evidence of significant radiation damage in this ultrasmall LB crystal [28]. The photoelectron escape from a narrow diffraction channel is a possible reason for reduced radiation damage as indicated by Monte Carlo simulations. These results open many new opportunities in scanning protein microcrystallography and make random data collection from microcrystals a real possibility, thereby enabling structures to be solved from much smaller crystals than previously anticipated as long as the crystallites are LB prepared. Data sets from several LB and classical crystals were collected to choose the best diffracting crystals for complete analysis. Typical classical crystal dimensions were $100 \times 200 \times 150$, and LB crystal dimensions were $100 \times 300 \times 150 \mu \mathrm{m}$. Beam size used for analysis was $100 \times 100 \mu \mathrm{m}$. The total dose absorbed by the two crystals during burning and normal data collection corresponds to 9.5 MGy. Data collection statistics for before and after burning of LB and classical crystals are presented in reference [26]. In order to study radiation damage in detail, electron-density maps were compared for first and last data sets for classical and LB-based crystals. The most characteristic structural effect of irradiation is electron density loss between disulfide bonds and carboxylic acid groups of aspartic and glutamic acid residues $[13,26]$. Thus, electron densities for some of these residues are compared before and after the burning process. The average B-factor indicates the positional spread of each atom in the protein after burning; the increase for each residue is more significant for classical crystals compared with LB-based crystals, indicating more damage in classical crystals. Overall analysis indicates that more damage is suffered by classical crystals compared with LB-based crystals. Loss of electron density at the same contour levels and increase in average B-factors clearly indicates that radiation damage is more significant in classical-based crystals compared with LB-based crystals.

\section{Conclusion}

The combination of the four label-free NAPPA technologies and DNASER fluorescent technology in conjunction with bioinformatics and protein nanobiocrystallography can be effectively used to quantitatively probe the expression and the atomic 3D structure of key proteins involved in the control of the mammalian cell cycle and cell transformation, thus also allowing the identification of query proteins for NAPPA and/or design of specific NAPPA microarrays. The results obtained and the work in progress indicate that the application of NAPPA may have provided deeper insights into cellular processes than could have been expected when it was first developed. Namely, instead of studying details of protein interactions of specific protein subsets/cascades, opportunities are now provided to study processes at the cellular level, for which only genomic (generally abundance-based) approaches used to be available. Label-free NAPPA technology, in combination with protein nanobiocrystallography and its possible future development using anodic porous alumina along with a cellfree expression system (as specified in [29] and summarized here in Figure 3), appear to form a single approach capable of effectively solving the numerous problems still present in medical diagnosis and therapy.

Financial \& competing interests disclosure

This work was supported by two FIRB Grants from MIUR (Italy) to CIRSDNNOB at the University of Genova, namely one on functional proteomics and cell cycle and the other one on organic and biological nanosensors, and by Funzionamento Grant 2008-2010 from MIUR (Italy) to Fondazione El.B.A. (Rome). The authors have no other relevant affliations or financial involvement with any organization or entity with a financial interest in or financial conflict with the subject matter or materials discussed in the manuscript apart from those disclosed.

No writing assistance was utilized in the production of this manuscript. 


\section{Bibliography}

1 Nicolini C: Nanogenomics for medicine. Nanomedicine 1, 147-151 (2006).

2 Nicolini C: Nanogenomics in medicine. Wiley Interdiscip. Rev. Nanomed. Nanobiotechnol. 2, 59-76 (2010).

3 LaBaer J, Ramachandran N: Protein microarrays as tools for functional proteomics. Curr. Opin. Chem. Biol. 9, 14-19 (2005).

4 Nicolini C, LaBaer J: Functional Proteomics and Nanotechnology-Based Microarrays (Volume 2). Nicolini C, LaBaer J (Eds). Pan Stanford Series on Nanobiotechnology, London-New York-Singapore, 1-345 (2010).

5 Sivozhelezov V, Giacomelli L, Tripathi S, Nicolini C: Gene expression in the cell cycle of human T lymphocytes: I. Predicted gene and protein networks. J. Cell. Biochem. 97, 1137-1150 (2006).

6 Sivozhelezov V, Spera R, Giacomelli L et al.: Bioinformatics and fluorescence DNASER for NAPPA studies on cell transformation and cell cycle. In: Functional Proteomics and Nanotechnology-Based Microarrays (Volume 2). Nicolini C, LaBaer J (Eds). Pan Stanford Series on Nanobiotechnology, London-New York-Singapore, 31-59 (2010).

7 Sartore M, Bezerra T, Eggenhöffner R et al.: Label free detection of NAPPA: atomic force microscopy. In: Functional Proteomics and Nanotechnology-Based Microarrays (Volume 2). Nicolini C, LaBaer J (Eds). Pan Stanford Series on Nanobiotechnology, London-New York-Singapore, 109-120 (2010).

8 Adami M, Eggenhöffner R, Sartore S, Hainsworth G, LaBaer J, Nicolini C: Label free NAPPA via nanogravimetry. In: Functional Proteomics and NanotechnologyBased Microarrays (Volume 2). Nicolini C, LaBaer J (Eds). Pan Stanford Series on Nanobiotechnology, London-New York-Singapore, 79-93 (2010).

9 Pechkova E, Nicolini C: Protein nanocrystallography: a new approach to structural proteomics. Trends Biotechnol. 22, 117-122 (2004).

10 Pechkova E, Nicolini C: Proteomics and Nanocrystallography. Kluwer Academic Publishers, 1-212 (2003).
11 Pechkova E, Sivozhelezov V, Tropiano G, Fiordoro S, Nicolini C: Comparison of lysozyme structures derived from thin-film-based and classical crystals. Acta Crystallogr. D Biol. Crystallogr. 61, 803-808 (2005).

12 Ravelli RBG, Leiros HKS, Pan BC, Caffrey M, McSweeney S: Specific radiation damage can be used to solve macromolecular crystal structures. Structure 11, 217-224 (2003).

13 Pechkova E, McSweeney S, Nicolini C: Atomic structure and radiation resistance of Langmuir-Blodgett protein crystals. In: Synchrotron Radiation and Structural Proteomics (Volume 3). Riekel C, Pechkova E (Eds). Pan Stanford Series on Nanobiotechnology, London-New YorkSingapore, 249-275 (2010)

14 Volkringer C, Popov D, Loiseau T et al.: A microdiffraction set-up for nanoporous metal-organic-framework-type solids. Nat. Mater. 6, 760-764 (2007).

15 Moukhametzianov R, Burghammer M, Edwards PC et al.: Protein crystallography with a micrometre-sized synchrotronradiation beam. Acta Crystallogr. D Biol. Crystallogr. 64, 158-166 (2008).

16 Pechkova E, Riekel C, Nicolini C: Growth and organization of LB protein crystals via in situ GISAXS, LASER, nanodiffraction, Raman and AFM. In: Synchrotron Radiation and Structural Proteomics (Volume 3). Riekel C, Pechkova E (Eds). Pan Stanford Series on Nanobiotechnology, London-New York-Singapore, 1-406 (2010).

17 Ravelli RBG, McSweeney SM: The 'fingerprint' that $\mathrm{x}$-rays can leave on structures. Structure 8, 315-328 (2000).

18 Pechkova E, Tropiano G, Riekel C, Nicolini C: Radiation stability of protein crystals grown by nanostructured templates: synchrotron microfocus analysis. Spectrochim. Acta B 59, 1687-1693 (2004).

19 Burmeister WP: Structural changes in a cryo-cooled protein crystal owing to radiation damage. Acta Crystallogr. D Biol. Crystallogr. 56, 328-341 (2000).
20 Stura E, Larosa C, Bezerra Correia Terencio T et al:: Label-free NAPPA: anodic porous alumina. In: Functional Proteomics and Nanotechnology-Based Microarrays (Volume 2). Nicolini C, LaBaer J (Eds). Pan Stanford Series on Nanobiotechnology, London-New York-Singapore, 95-108 (2010).

21 Nicolini C, Malvezzi M, Tomaselli A, Sposito D, Tropiano G, Borgogno E: DNASER I: layout and data analysis. IEEE Trans. Nanobioscience 1, 67-72 (2002).

22 Nicolini C, Spera R, Stura E, Fiordoro S, Giacomelli L: Gene expression in the cell cycle of human T lymphocytes: II. Experimental determination by DNASER technology. J. Cell. Biochem. 97, 1151-1159 (2006).

23 Nicolini C, Pechkova E: An overview of nanotechnology-based functional proteomics for cancer and cell cycle progression. Anticancer Res. (2010) (In Press).

24 Nicolini C: Protein monolayer engineering: principles and application to biocatalysis Trends Biotechnol. 15, 395-401 (1997).

25 Nicolini C, Pechkova E: Nanostructured biofilms and biocrystals. J. Nanosci. Nanotechnol. 6, 2209-2236 (2006).

26 Pechkova E, Tripathi S, Ravelli R, McSweeney S, Nicolini C: Radiation stability of proteinase $\mathrm{K}$ grown by LB nanotemplate method. J. Struct. Biol. 168, 409-418 (2009).

27 Pechkova E, Sartotre M, Giacomelli L, Nicolini C: Atomic force microscopy of protein films and crystals. Rev. Sci. Instrum. 78, 093704 (2007).

28 Pechkova E, Nicolini C: Domains organization and properties of LB lysozyme crystals down to submicron size. Anticancer Res. (2010) (In Press).

29 Pechkova E, Chong R, Tripathi S, Nicolini C: Cell free expression and APA for NAPPA and protein nanocrystallography. In: Functional Proteomics and NanotechnologyBased Microarrays (Volume 2). Nicolini C, LaBaer J (Eds). Pan Stanford Series on Nanobiotechnology, London-New YorkSingapore, 121-147 (2010). 